\title{
Early Experience of Direct Anterior Approach Total Hip Arthroplasty: Analysis of the First 53 Cases
}

\author{
Chung-Young Kim, MD, Young-Yool Chung, MD, Seung-Woo Shim, MD, \\ Sung-Nyun Baek, MD, Cheol-Hwan Kim, MD \\ Department of Orthopaedic Surgery, Kwangju Christian Hospital, Gwangju, Korea
}

Purpose: To determine if it is feasible and safe for a surgeon to transition from using the posterolateral approach to direct anterior approach (DAA) by evaluating the first 53 cases of total hip arthroplasty using a DAA. Materials and Methods: A retrospective review of 52 patients who underwent THA using a DAA between July 2017 and December 2018. Reasons for THA were: femoral neck fracture $(n=34)$, avascular necrosis $(n=13)$, and arthritis $(n=6)$. The mean age was 70 years old. An assessment of feasibility was made by analyzing mean operative time and blood loss. Cup inclination, anteversion, and leg length discrepancy (LLD) were measured using postoperative radiology. Safety of the DAA was judged using the incidence and nature of all complications.

Results: The mean operative time was 112 minutes. 135 minutes for the 1 st 10 cases, 100 minutes for 2 nd 10 cases, 113 minutes for 3rd 10 cases, 119 minutes for 4th 10 cases, and 91 minutes for the final 13 cases. The mean blood loss was $724 \mathrm{~mL}$. Average cup inclination was $40.27^{\circ} ; 2$ cases were out of safety angle. Mean anteversion was $16.18^{\circ}$. No intraoperative fractures or infections were observed. LLD was detected in 3 cases, one of which underwent revision due to walking difficulty. Dislocation occurred in 3 cases, all within the first 20 cases, however, there was no recurrent dislocation.

Conclusion: DAA for THA was deemed to be feasible and safe based on an assessment of operative time, blood loss and complications.

Key Words: Hip replacement arthroplasty, Learning curve, Minimal invasive surgery

Submitted: September 30, 2019 1st revision: December 6, 2019

2nd revision: January 30, 2020 3rd revision: March 4, 2020

Final acceptance: March 5, 2020

Address reprint request to

Young-Yool Chung, MD

(https://orcid.org/0000-0002-4959-1230)

Department of Orthopaedic Surgery, Kwangju Christian Hospital,

37 Yangrim-ro, Nam-gu, Gwangju 61661, Korea

TEL: +82-62-650-5064 FAX: +82-62-650-5066

E-mail: wonderchunglanaver.com

This is an Open Access article distributed under the terms of the Creative Commons Attribution Non-Commercial License (http://creativecommons. org/licenses/by-nc/4.0) which permits unrestricted non-commercial use, distribution, and reproduction in any medium, provided the original work is properly cited.

\section{INTRODUCTION}

Along with advances in implant materials and surgical techniques for total hip arthroplasty (THA), favorable results at long-term follow-up have been reported and indications for the procedure have expanded. In recent years, investigations on surgical procedures that reduce postoperative pain and improve recovery times by minimizing soft tissue injuries have continued.

A variety of surgical approaches for THA have been introduced; the posterolateral approach (PA) is the most common ${ }^{1)}$. However, PA has been associated with increased soft tissue injury and higher dislocation rates compared to other approaches ${ }^{2)}$. 
In 2003, Mears ${ }^{3)}$ introduced two-incision minimally invasive THA that minimizes soft tissue damage, enables early postoperative ambulation, and shortens hospital stays. However, this technique has not been adopted as a common surgical intervention due to a long learning curve and a high incidence of early postoperative complications. The direct anterior approach (DAA) for THA has been garnering much attention because the procedure matches the aim of minimally invasive surgical procedures ${ }^{4,5)}$.

Some of the benefits of DAA compared with other approaches include less damage to soft tissue, reduced postoperative pain with no muscle splitting or injury, rapid recovery of gait ability, more stable artificial hip and decreased dislocation risk. Moreover, this approach allows easier prediction of implant position and facilitates the use of fluoroscopic imaging during surgery since the patient is in the supine position ${ }^{6,7}$. Despite these advantages, DAA requires longer operative time, is associated with a long learning curve as it is technically demanding, and has been shown to be associated with high rates of complications (e.g., proximal femoral fracture, infection), ${ }^{2,8-10)}$.

The authors of this study aimed to determine the feasibility and safety for a surgeon to transition from PA to DAA THA by assessing operation times and early complications in the first 53 cases of THA using the DAA at a single institution.

\section{MATERIALS AND METHODS}

\section{Subjects}

This study included a review of data from 52 patients (53 cases; 15 males and 38 females) who underwent THA using a DAA between June 2017 and December 2018. All operations were performed by a senior author who had experience performing more than 500 THAs using a PA. The mean patient age at time of surgery was 70 years old (range, 26-94 years), mean BMI was $23.06 \mathrm{~kg} / \mathrm{m}^{2}$ (range, $17.30-30.44 \mathrm{~kg} / \mathrm{m}^{2}$ ) and mean BMD was -2.9 $\mathrm{g} / \mathrm{cm}^{2}$ (range, -5.8 to $-1.0 \mathrm{~g} / \mathrm{cm}^{2}$ ). BMD was the average of two lowest T-scores from L1 to L4 or the lowest Tscore obtained from either the contralateral femoral neck or total femur. Preoperative diagnosis revealed 34 cases of femoral neck fracture, 13 cases of avascular necrosis of the femoral head and 6 cases of osteoarthritis. The acetabular component used was a Bencox ${ }^{\mathbb{R}}$ cup (Corentec, Cheonan, Korea) $(\mathrm{n}=48)$, and a Continuum ${ }^{\circledR}$ acetabular cup (Zimmer, Warsaw, IN, USA) (n=5). The femoral component was a Bencox ${ }^{\mathbb{R}} \mathrm{M}$ stem (Corentec) $(\mathrm{n}=48)$, and the $\mathrm{M} / \mathrm{L}$
Taper Hip Prosthesis (Zimmer) ( $\mathrm{n}=5$ ). Neither C-arms nor cement were used in the insertion of acetabular and femoral components.

\section{Surgical Methods}

All patients were positioned supine on a specialized orthopedic table (Hana ${ }^{\circledR}$ Orthopedic Fracture/Trauma Surgical Table; Mizuho OSI, Union City, CA, USA). After the anterior superior iliac spine was checked, the tensor fascia latae was palpated and incised, and then capsulotomy was performed via a medial approach without excision of tendons or muscles. The femoral neck was cut in twice and after removal of the femoral head, the acetabulum was exposed. After reaming by progressively increasing reamer diameters, the acetabular cup was inserted followed by placement of the liner. A femur elevator hook was placed around the proximal femur and then lifted up it with the leg externally rotated. To allow for femoral preparation, the hip was extended and adducted by lowering the foot-end of the Hana table. After determining the correct positioning and orientation of the femoral component using a canal finder, rasping was conducted and an appropriately sized stem was inserted. After impacting the head onto the stem, reduction was performed with proper traction and internal rotation in a leg-raising manner. After cleansing the surgical site and soft tissue wound, subcutaneous and skin layers were closed (Fig. 1).

\section{Study Methods}

For assessment of surgical procedures, the mean operative time and blood loss were measured. Operative time was defined by duration from skin incision to the completion of wound suturing. Total blood loss was defined as the sum of intraoperative blood loss and postoperative drainage volume.

For radiologic assessment, inclination of the acetabular component was measured on the anteroposterior pelvic radiograph taken immediately after surgery, and anteversion was measured on the cross-table lateral view. Leg length discrepancy was determined by measuring the distance between the ischial tuberosities and the superior margin of the lesser trochanter on AP pelvic radiographs taken with both legs internally rotated $15^{\circ}$. Leg-length discrepancy was considered to have occurred when the difference between the distances was greater than $10 \mathrm{~mm}$.

Intra- and postoperative complications that patients 


\section{Hip \& Pelvis}

Hip Pelvis 32(2): 78-84, 2020

experienced were examined (e.g., dislocation, proximal femoral fracture, infection).

This study was performed after gaining Institutional Review Board (IRB) approval from Kwangju Christian Hospital (IRB No. KCH-M-2019-03-007).

For statistical analysis, operative time and blood loss were analyzed in groups of 10 consecutive cases and a significant decrease was noted in the cut-off point. All statistical analyses were performed using PASW Statistics ver. 18.0 (IBM Corp., Armonk, NY, USA); chi-squared and paired $t$-tests were conducted to test for differences between two groups. Differences were considered statistically significant at $P<0.05$.
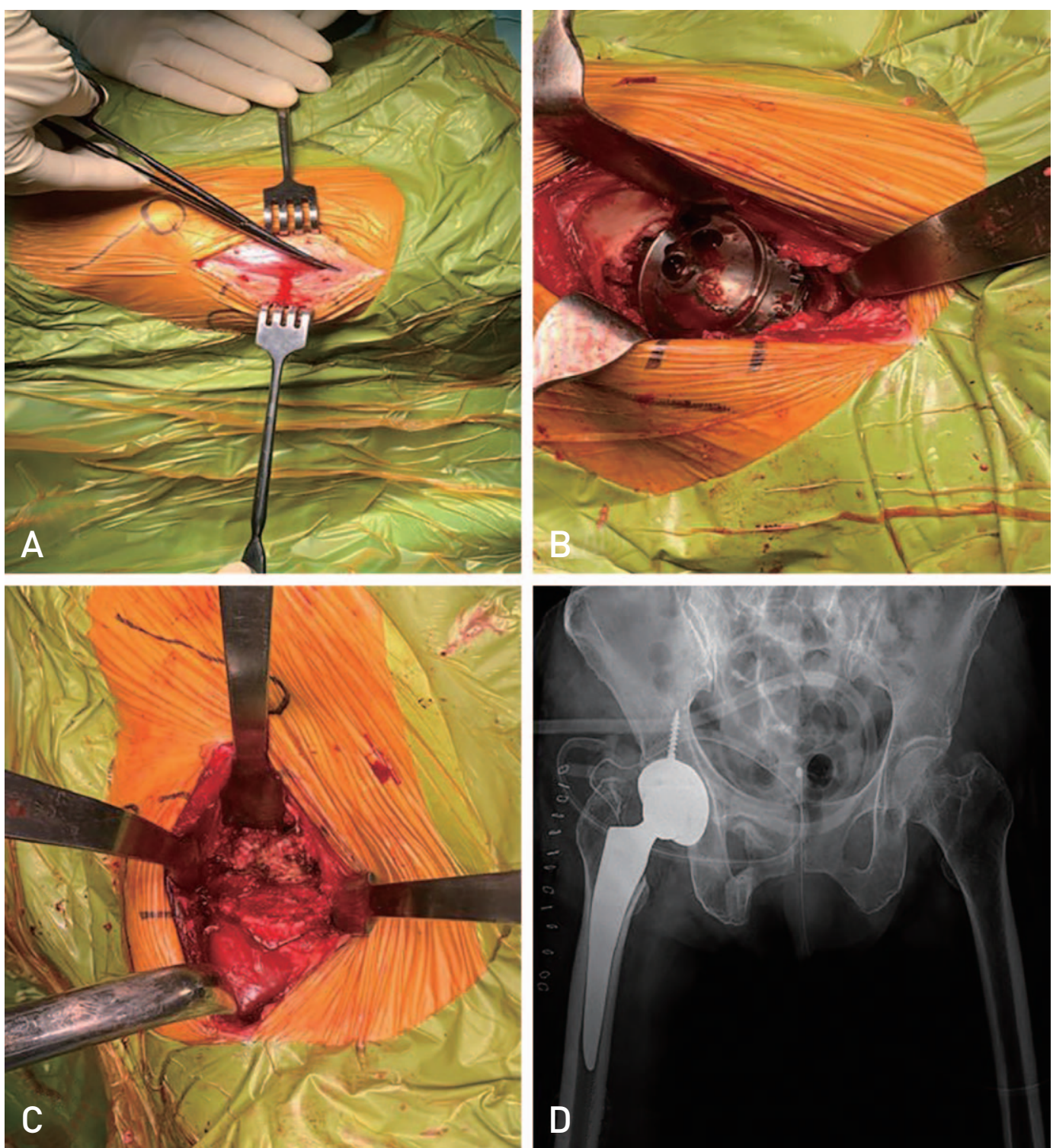

Fig. 1. (A) Direct anterior approach (DAA) skin incision; (B) Cup insertion, (C) Femur elevation and exposure; (D) Total hip arthroplasty using DAA was performed. 


\section{Hip \& Pelvis}

\section{Chung-Young Kim et al. Early Experience of DAA THA}

(Table 2).

The average cup inclination was $40.27^{\circ}$ (range, 23.8-55.1 $1^{\circ}$ ), and the cup was positioned outside of the Lewinnek et al. ${ }^{11)}$ safe zone in two cases (Fig. 2). The average cup anteversion was $16.18^{\circ}$ (range, $-2.41-52.3^{\circ}$ ). After the first 20 cases, there were significantly fewer $(n=0)$ anteversion angles outside the safe zone (Fig. 3).

No postoperative complications (e.g., infection, femoral fracture) were observed. A discrepancy in leg lengths more than $10 \mathrm{~mm}$ was detected in 3 cases $(5.7 \%)$; in one of these cases, revision surgery was conducted due to a complaint of discomfort when walking immediately after surgery. Anterior dislocation occurred in $3(5.7 \%)$ out of the first 20 cases; all were managed with conservative treatment and there were no recurrent dislocations.

Table 1. Comparison of Mean Operative Time according to the Serial Case Number of Surgeries

\begin{tabular}{lcc}
\hline \hline Number of case & Mean operation time (min) & $P$-value \\
\hline Initial group $(n=10): 2 n d$ group $(n=43)$ & $135 \pm 39: 104 \pm 21$ & 0.037 \\
Initial group $(n=20): 2 n d$ group $(n=33)$ & $117 \pm 35: 105 \pm 22$ & 0.151 \\
Initial group $(n=30): 2 n d$ group $(n=23)$ & $115 \pm 31: 103 \pm 21$ & 0.084 \\
Initial group $(n=40): 2 n d$ group $(n=13)$ & $116 \pm 29: 90 \pm 11$ & 0.003 \\
\hline
\end{tabular}

Values are presented as mean \pm standard deviation.

Table 2. Comparison of Mean Amount of Blood Loss according to the Serial Case Number of Surgeries

\begin{tabular}{lcc}
\hline \hline Number of case & Mean blood loss $(\mathrm{mL})$ & $P$-value \\
\hline Initial group $(\mathrm{n}=10): 2 \mathrm{nd}$ group $(\mathrm{n}=43)$ & $1,071 \pm 426: 643 \pm 197$ & 0.001 \\
Initial group $(\mathrm{n}=20): 2$ nd group $(\mathrm{n}=33)$ & $833 \pm 412: 657 \pm 189$ & 0.039 \\
Initial group $(\mathrm{n}=30): 2$ nd group $(\mathrm{n}=23)$ & $766 \pm 368: 668 \pm 176$ & 0.264 \\
Initial group $(\mathrm{n}=40): 2 \mathrm{nd}$ group $(\mathrm{n}=13)$ & $760 \pm 326: 616 \pm 180$ & 0.046 \\
\hline
\end{tabular}

Values are presented as mean \pm standard deviation.

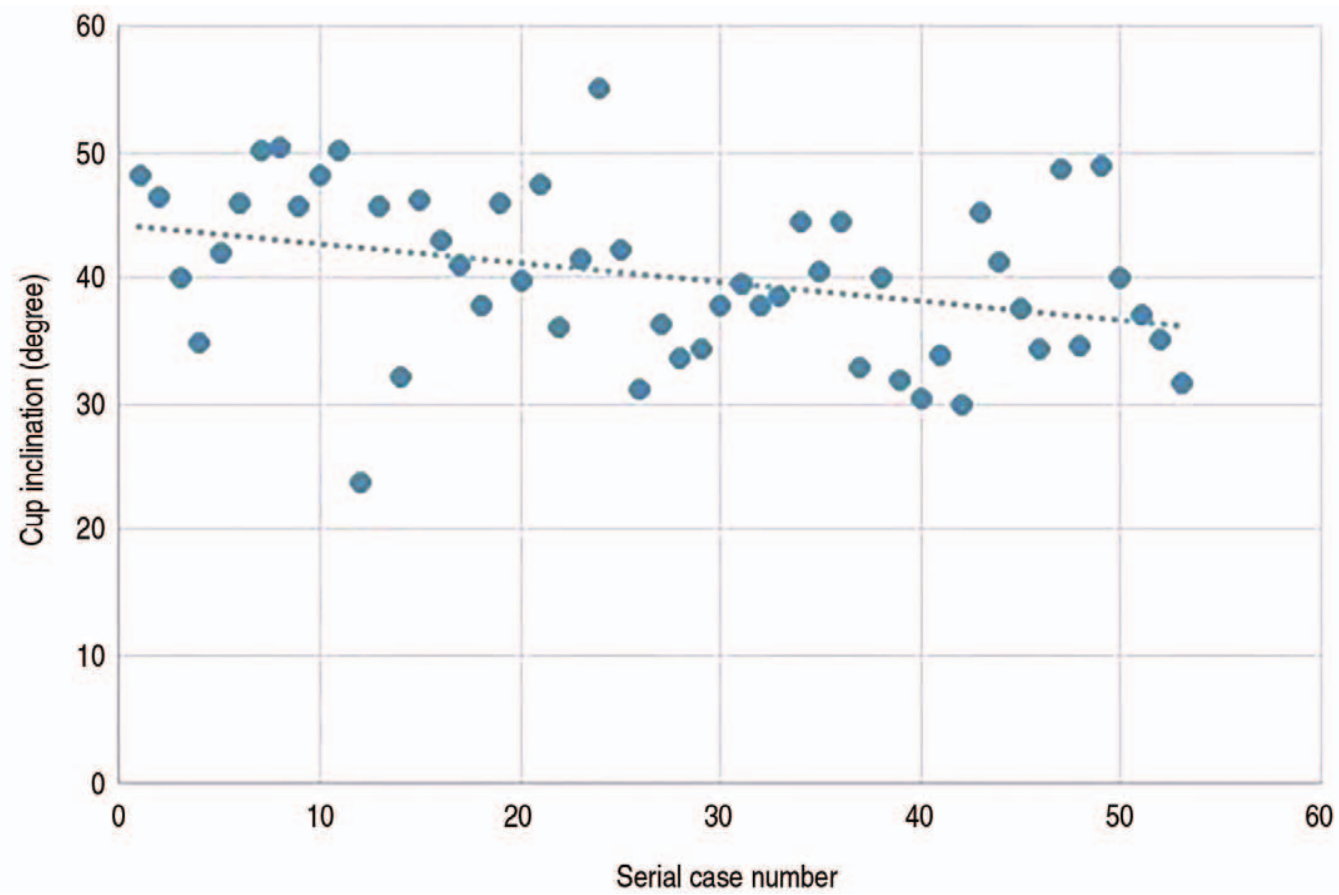

Fig. 2. Distribution of cup inclination - The average of cup inclination was $40.27^{\circ}$. Only 2 cases were out of Lewinnek's safety angle. 


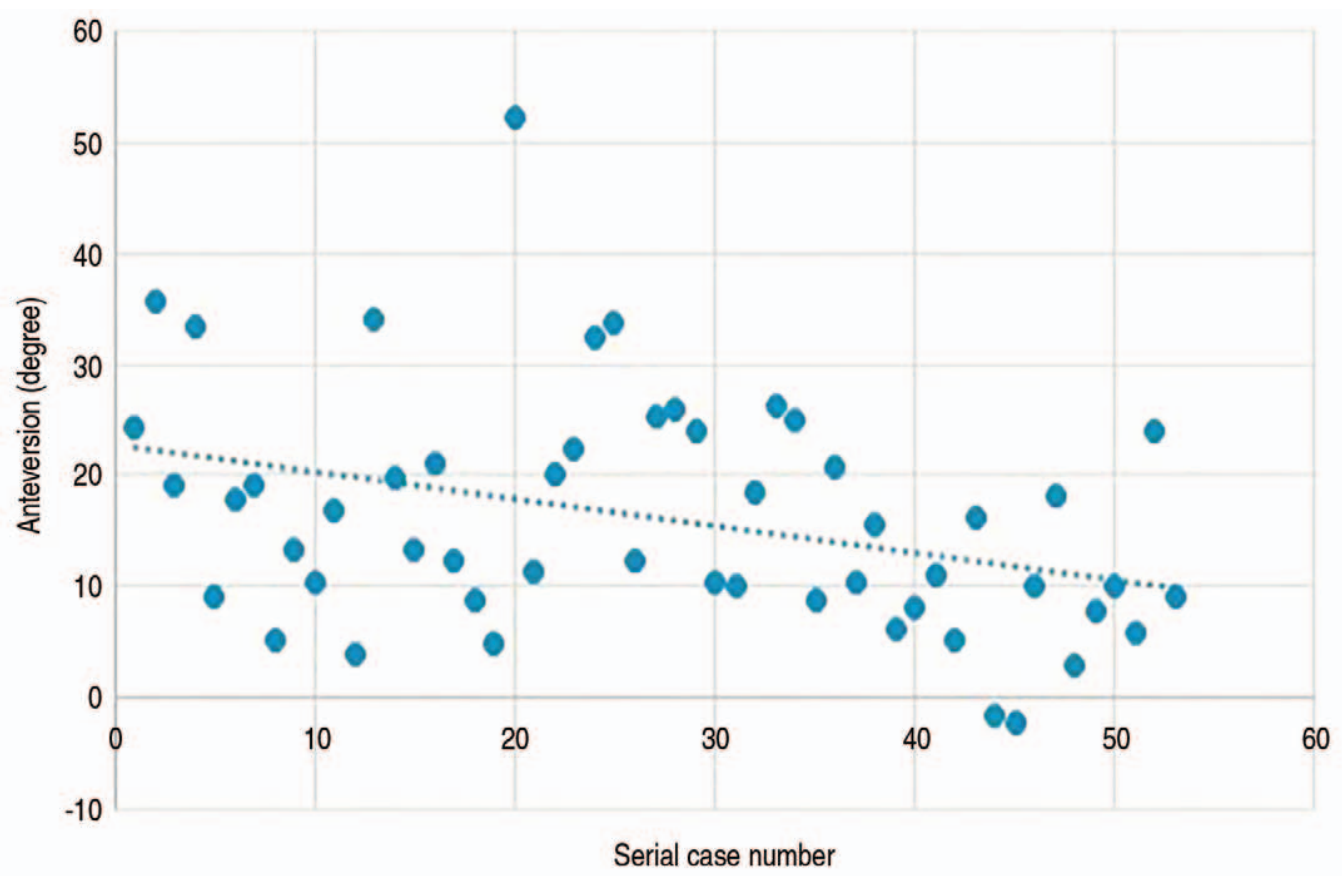

Fig. 3. Distribution of anteversion - The average of anteversion was $16.18^{\circ}$. After having conducted 20 surgeries, the variation decreased and fewer cases were outside of the Lewinnek's safty angle.

\section{DISCUSSION}

Refinements of prosthetic fixation and bearing surfaces for THA have increased survival rates for artificial hip joints as demonstrated by long-term follow-up studies. In recent years, surgical techniques that minimize damage to soft tissue and reduce postoperative pain, allowing for an early return to activities of daily living have increasingly gained much interest. In the early 2000s, two-incision minimally invasive THA was introduced with the goal of reducing damage to soft tissue. However, prolonged surgical time, increased blood loss and a high incidence of early postoperative complications have been reported due to the associated technical challenges. Tanavalee et al. ${ }^{12)}$ reported that twoincision THA was associated with problems including prolonged operative time and excessive blood loss. In a study by these authors ${ }^{13}$, the mean operative time was 163 minutes, mean volume of blood loss was $974 \mathrm{~mL}$, and intraoperative fracture occurred in 4 cases. Due to these limitations, two-incision THA has not been adopted as a common surgical procedure. As minimally invasive surgical procedures have gained increasing attention in recent years, the DAA has been the subject of many investigations ${ }^{5,899}$. In terms of operative time, blood loss and complication rates, multiple studies have reported the learning curve for DAA THAs ranges from 10 to 200 cases $^{14}$. In a study by Stone et al. ${ }^{15)}$ in a consecutive series of 1,000 cases when transitioning from PA to DAA THA, the mean operative time increased by about $30 \%$ in the initial 50 cases, and decreased by $14 \%$ in the second 50 cases. They reported that operative time of DAA THA was shorter compared with posterior hip arthroplasty after the 500th cases. Spaans et al.9) reported that the mean surgical time decreased with increasing experience in 46 DAA THA cases. In our study, the mean surgical time was 135 minutes in the first 10 cases, decreased to 104 minutes $(23 \%$ reduction), and reduced to 90.7 minutes (an additional 13\% reduction) after the 40th cases. In the authors' study on two incision THA ${ }^{3}$, surgical time exceed 2 hours in all but 3 of 26 cases, and it appears as though a steeper learning curve may exist as compared with DAA since the average surgical time was 104 minutes after the 10th cases of DAA THA. Similar to two-incision THA, the major disadvantage of DAA has been reported to be an increase in blood loss. In a comparative study on DAA versus PA THA by Spaans et al. ${ }^{9}$, the mean intraoperative blood loss was $703 \mathrm{~mL}$ in the DAA group, higher when compared to the PA group $(364 \mathrm{~mL})$, and there was no apparent reduction in the learning curve. Barnett et al. ${ }^{16)}$ assessed the overall volume of intra- and postoperative blood loss in 5,090 consecutive THA cases using the DAA. The mean blood loss was less than $400 \mathrm{~mL}$ in $67.1 \%$ and $400-800 \mathrm{~mL}$ in $29.4 \%$. In this 
study, the overall average blood loss was $724 \mathrm{~mL}$. This average was $1,071 \mathrm{~mL}$ in the first 10 cases, $643 \mathrm{~mL}$ (reduction of $40 \%$ ) in the remaining cases. Importantly, the average blood volume loss was $612 \mathrm{~mL}$ after the 40th case. Blood loss was dramatically reduced as additional experience was gained $^{17)}$.

Since DAA THA is typically performed with patients in the supine position, a position familiar to surgeons, this approach allows for easier prediction and control when positioning acetabular and femoral components accurately and facilitates the use of intraoperative fluoroscopy ${ }^{18}$. A radiologic assessment of DAA vs. PA THA by Cheng et al. ${ }^{19)}$ reported that average inclination and anteversion angles of the acetabular cup were similar, however, the mean cup inclination and anteversion were within the Lewinnek safe zone (inclination: $40 \pm 10^{\circ}$; anteversion: $\left.15 \pm 10^{\circ}\right)^{11)}$ in $57 \%$ and $34 \%$ of DAA and PA cases, respectively. In a comparison of inclination and anteversion in 100 THA cases each by DAA and Hamilton et al. ${ }^{20)}$ noted that anteversion angles were different between those treated with DAA $\left(17.6^{\circ}\right)$ compared with those treated with PA $\left(22.6^{\circ}\right)$; inclination angles were similar in both approaches, however, it was reported that there was less variation in the DAA group. Although fluoroscopic imaging was not used in the current study, the average cup inclination was $40.27^{\circ}$, and the cup was positioned outside of the Lewinnek safe zone in only two cases. The average cup anteversion was $16.18^{\circ}$. Variation in anteversion was reduced after the first 20 cases, cases which the cup was positioned outside of the Lewinnek safe zone have also been reduced. Despite several advantages, the DAA has been reported to result in increased complication rates $^{9,21}$. There are risks of intraoperative fracture due to traction and difficulty with complete exposure of the proximal femur, and hypoesthesia and paresthesia on the lateral femur due to injuries to the lateral femoral cutaneous nerve ${ }^{9,10}$. In are view by Barnett et al. ${ }^{16}$ involving 5,090 consecutive THA cases using the DAA, intraoperative fractures were reported in 43 cases, calcar fracture in 26 , and greater trochanteric fracture in 12 . In the current study, no proximal femur fractures were detected. The use of the Hana fracture table that allows for lifting-up safely using femur elevate hook and for safe hyperextension, adduction and external rotation of the leg improved exposure of the greater trochanter is thought to prevent intraoperative fractures.

Theoretically, preservation of posterior structures (e.g., posterior capsule, abductors) have been shown to reduce dislocation rates. In previous studies, postoperative dislocation rates ranged between 0.6 and $1.2 \%{ }^{22,23)}$. Free et al. ${ }^{24)}$ stated that dislocation did not occur in the initial 93 cases when transitioning to DAA THA and recommended that the DAA is a safe approach for novice surgeons. However, dislocations occurred in 3 of the first 20 cases in this study. Dislocation is thought to be caused by extensive resection of soft tissue for improved exposure of the proximal femur in the initial patients. There were no dislocations after the 20th case.

There are some limitations to note in the present study. First, there is a relatively short follow-up period due to its retrospective nature. This study was also limited by no clinical assessment of possible injuries (e.g., lateral femoral cutaneous nerve injury). Additional studies are warranted to further elucidate complications and prognoses. Despite these limitations, this study will be meaningful for surgeons who intend to transition from PA THA to DAA THA by highlighting the early experience of surgeons (i.e., the authors of this study) who are more familiar with PA THA. Importantly, the findings of this study cannot be generalized due to the relatively small sample size. Second, more studies with a larger sample size are warranted. In particular, the results from this study cannot be generalized because most subjects were elderly patients with femoral neck fractures. Third, prospective studies comparing PA and other approaches are thought to be needed. Furthermore, additional studies on the cut-off point with improved safety after switching to the DAA are warranted.

\section{CONCLUSION}

The DAA for THA is an alternative procedure that may replace the convention surgical approach by reducing the mean operative time and blood loss in a short learning curve. The risk of complications was not as high as expected. Caution is required to prevent possible complications from DAA as is the case for any new surgical technique.

\section{CONFLICT OF INTEREST}

The authors declare that there is no potential conflict of interest relevant to this article.

\section{REFERENCES}

1. Kurtz S, Ong K, Lau E, Mowat F, Halpern M. Projections of primary and revision hip and knee arthroplasty in the United States from 2005 to 2030. J Bone Joint Surg Am. 2007;89:780-5.

2. De Geest T, Vansintjan P, De Loore G. Direct anterior total hip arthroplasty: complications and early outcome in a 
series of 300 cases. Acta Orthop Belg. 2013;79:166-73.

3. Mears DC. Development of a two-incision minimally invasive total hip replacement. J Bone Joint Surg Am. 2003;85: 2238-40.

4. Chechik O, Khashan M, Lador R, Salai M, Amar E. Surgical approach and prosthesis fixation in hip arthroplasty world wide. Arch Orthop Trauma Surg. 2013;133:1595-600.

5. Kennon RE, Keggi JM, Wetmore RS, Zatorski LE, Huo $\mathrm{MH}$, Keggi KJ. Total hip arthroplasty through a minimally invasive anterior surgical approach. J Bone Joint Surg Am. 2003;85 Suppl 4:39-48.

6. Rachbauer F, Kain MS, Leunig M. The history of the anterior approach to the hip. Orthop Clin North Am. 2009;40:311-20.

7. Sibia US, Turner TR, MacDonald JH, King PJ. The impact of surgical technique on patient reported outcome measures and early complications after total hip arthroplasty. $J$ Arthroplasty. 2017;32:1171-5.

8. Eto S, Hwang K, Huddleston JI, Amanatullah DF, Maloney WJ, Goodman SB. The direct anterior approach is associated with early revision total hip arthroplasty. J Arthroplasty. 2017;32:1001-5.

9. Spaans AJ, van den Hout JA, Bolder SB. High complication rate in the early experience of minimally invasive total hip arthroplasty by the direct anterior approach. Acta Orthop. 2012;83:342-6.

10. Goulding K, Beaulé PE, Kim PR, Fazekas A. Incidence of lateral femoral cutaneous nerve neuropraxia after anterior approach hip arthroplasty. Clin Orthop Relat Res. 2010; 468:2397-404.

11. Lewinnek GE, Lewis JL, Tarr R, Compere CL, Zimmerman JR. Dislocations after total hip-replacement arthroplasties. J Bone Joint Surg Am. 1978;60:217-20.

12. Tanavalee A, Jaruwannapong S, Yuktanandana P, Itiravivong P. Early outcomes following minimally invasive total hip arthroplasty using a two-incision approach versus a miniposterior approach. Hip Int. 2006;16 Suppl 4:17-22.

13. Ki SC, Kim BH, Ryu JH, Yoon DH, Chung YY. Total hip arthroplasty using two-incision technique. Clin Orthop Surg. 2011;3:268-73.

14. den Hartog YM, Mathijssen NM, Vehmeijer SB. The less invasive anterior approach for total hip arthroplasty: a comparison to other approaches and an evaluation of the learning curve - a systematic review. Hip Int. 2016;26:10520.

15. Stone AH, Sibia US, Atkinson R, Turner TR, King PJ. Evaluation of the learning curve when transitioning from posterolateral to direct anterior hip arthroplasty: a consecutive series of 1000 cases. J Arthroplasty. 2018;33:2530-4.

16. Barnett SL, Peters DJ, Hamilton WG, Ziran NM, Gorab RS, Matta JM. Is the anterior approach safe? Early complication rate associated with 5090 consecutive primary total hip arthroplasty procedures performed using the anterior approach. J Arthroplasty. 2016;31:2291-4.

17. Lee SH, Kang SW, Jo S. Perioperative comparison of hip arthroplasty using the direct anterior approach with the posterolateral approach. Hip Pelvis. 2017;29:240-6.

18. Matta JM, Shahrdar C, Ferguson T. Single-incision anterior approach for total hip arthroplasty on an orthopaedic table. Clin Orthop Relat Res. 2005;441:115-24.

19. Cheng TE, Wallis JA, Taylor NF, Holden CT, Marks P, Smith CL, et al. A prospective randomized clinical trial in total hip arthroplasty-comparing early results between the direct anterior approach and the posterior approach. $J$ Arthroplasty. 2017;32:883-90.

20. Hamilton WG, Parks NL, Huynh C. Comparison of cup alignment, jump distance, and complications in consecutive series of anterior approach and posterior approach total hip arthroplasty. J Arthroplasty. 2015;30:1959-62.

21. de Steiger RN, Lorimer M, Solomon M. What is the learning curve for the anterior approach for total hip arthroplasty? Clin Orthop Relat Res. 2015;473:3860-6.

22. Abdel MP, von Roth P, Jennings MT, Hanssen AD, Pagnano MW. What safe zone? The vast majority of dislocated THAs are within the Lewinnek safe zone for acetabular component position. Clin Orthop Relat Res. 2016;474:386-91.

23. Khatod M, Barber T, Paxton E, Namba R, Fithian D. An analysis of the risk of hip dislocation with a contemporary total joint registry. Clin Orthop Relat Res. 2006;447:19-23.

24. Free MD, Owen DH, Agius PA, Pascoe EM, Harvie P. Direct anterior approach total hip arthroplasty: an adjunct to an enhanced recovery pathway: outcomes and learning curve effects in surgeons transitioning from other surgical approaches. J Arthroplasty. 2018;33:3490-5. 